\title{
Ground State Properties of the Diluted Sherrington-Kirkpatrick Spin Glass
}

\author{
Stefan Boettcher ${ }^{\text {* }}$ \\ Physics Department, Emory University, Atlanta, Georgia 30322, USA
}

\begin{abstract}
We present a numerical study of ground states of the dilute versions of the Sherrington-Kirkpatrick (SK) mean-field spin glass. In contrast to so-called "sparse" mean-field spin glasses that have been studied widely on random networks of finite (average or regular) degree, the networks studied here are randomly bond-diluted to an overall density $p$, such that the average degree diverges as $\sim p N$ with the system size $N$. Ground-state energies are obtained with high accuracy for random instances over a wide range of fixed $p$. Since this is a NP-hard combinatorial problem, we employ the Extremal Optimization heuristic to that end. We find that the exponent describing the finite-size corrections, $\omega$, varies continuously with $p$, a somewhat surprising result, as one would not expect that gradual bond-dilution would change the $T=0$ universality class of a statistical model. For $p \rightarrow 1$, the familiar result of $\omega(p=1) \approx \frac{2}{3}$ for SK is obtained.
\end{abstract}

The Sherrington-Kirkpatrick model (SK) [1] was devised as the mean-field limit of finite-dimensional Ising spin glasses, first introduced by Edwards and Anderson (EA) 2], to describe the unusual phenomenology [3] of disorder in the interaction between classical dipolar magnets in certain materials. Despite the dramatic simplification that such a limit entails, i.e., replacing the lattice with a dense network of bonds between all mutual pairs of spins, SK proved so intricate that it took several years and a herculean effort by Parisi to reveal its full structure, referred to as replica symmetry breaking (RSB) [4 6 ]. RSB was verified rigorously only thirty years later [7, 8]. Over the years, the importance of these Ising spin glass models has significantly increased as a most concise conceptualization of systems with disorder and frustration, and the complex structure and dynamics that emerges [6. 9. Far beyond its origins in materials science, SK has inspired notions of learning in neural networks and artificial intelligence [10, actual neurons [11, facilitated optimization of hard combinatorial problems in operations research and engineering [6, 12, 14, elucidated the nature of energy landscapes [15], made connections to biological evolution [16], social dynamics [17], etc. Ironically, in most of these applications, the unstructured mean-field version of a glass, such as SK, is far more realistic than the lattice geometry of EA. Moreover, many of these problems, like optimization and learning, concern the low-temperature limit, instead of the physically pertinent phase transition at some finite critical temperature $T_{c}$ : As long as $T_{c}>0$, a glassy phase exists at $T \rightarrow 0$. In fact, in mean-field there is an entire critical line extending from $T_{c}$ to $T=0$ [18. Notably, $T=0$ is its own fixed point [19] in the renormalization group sense [20], with its own set of scaling relations, yet to be completed [21 25], connecting domain-wall excitations, ground-state energy fluctuations, and finite-size corrections.

Extending RSB to glassy systems on sparse networks, i.e., random graphs [26] of finite average or fixed degree ("Bethe lattices", BL), constituted another major breakthrough [27]. More recently, the one-dimensional longrange model [28] has gained popularity [29 32] for the

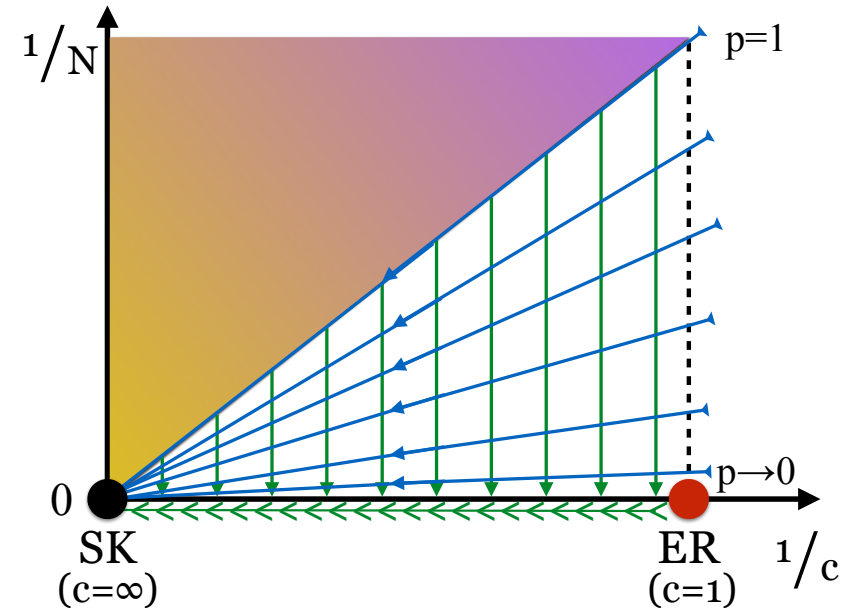

Figure 1: Depiction of alternative ways to approach the thermodynamic limit $N \rightarrow \infty$ (or, $1 / N \rightarrow 0$ ) for mean-field spinglass models of (average or fixed) spin-degree $c$. Previous work had been focused on constant $c$ while $1 / N \rightarrow 0$ (green downarrows ), referred to as "Bethe lattices" due to their locally tree-like structure [27. In Ref. 36, 37, it was shown that the thermodynamic limit of their ground-state energy densities $\left\langle e_{0}\right\rangle_{N=\infty}^{\text {Bethe }}$ can be connected (horizontal green arrows) to that of SK (black dot) via $\left\langle e_{0}\right\rangle_{N=\infty}^{\text {Bethe }} \sim c^{\frac{1}{2}} e_{\text {Parisi }}$, at least for $c \gg 1$, i.e., above the Erdös-Rényi percolation transition for sparse random graphs [26] (red dot). This study explores a diluted SK system, in which system size $N$ and connectivity $c$ both evolve such that $p \sim c / N$ remains constant (blue rays).

ability to interpolate between SK and the EA (but on a $1 d$-ring geometry) based on the range of interactions. That model has effective upper and lower dimensions, but all results obtained are numerical.

It is thus surprising that after so many years of studying mean-field spin glasses in the thermodynamic limit on fully connected (SK) or on sparse networks (BL), there has been no consideration given to dense but dilute systems. (Ref. [33, concerning optimal graph bipartitioning, a problem closely related to spin glasses [34], might pose a rare exception.) For BL, the average or fixed number $c$ of other spins that any one spin is randomly bonded 
with, i.e., its "degree", is held constant for all network sizes $N \rightarrow \infty$. In contrast, it is the average bond density,

$$
p=\frac{c}{N-1},
$$

that is held constant in a dilute system. Clearly, in SK each spin has a bond to every one of the other spins, i.e., $c_{\mathrm{SK}}=N-1$ and $p=1$, while at some general $0<p \leq 1$, the degree for each spin diverges as $c \sim p N$ in the thermodynamic limit $N \rightarrow \infty$. Thus, dilute SK presents a true alternative to BL, for which $p \sim 1 / N \rightarrow 0$, likely resulting in an alternative RSB analysis. These connections are illustrated in Fig. 1. In this Letter, we provide some tantalizing numerical evidence that such an analysis might be quite distinct and potentially more fruitful in revealing, for instance, the nature of finite-size corrections (FSC) that occur when $N \rightarrow \infty$, which have remained beyond the scope of RSB.

Understanding the nature of FSC for $N \rightarrow \infty$ is an essential ingredient in the proper interpretation of numerical data obtained from thermodynamic systems [35]. To reach the thermodynamic limit with data derived from, inevitably, finite-size simulations usually requires a certain degree of extrapolation 36 43. Here, we will specifically focus on FSC to the ensemble average of the ground state energy density, assuming the form

$$
\left\langle e_{0}\right\rangle_{N} \sim\left\langle e_{0}\right\rangle_{\infty}+\frac{A}{N^{\omega}}, \quad(N \rightarrow \infty),
$$

defining the energy density in the thermodynamic limit, $\left\langle e_{0}\right\rangle_{\infty}$. In many disordered systems, such as for spin glasses in the low-temperature limit exhibiting RSB, those FSC are dogged by (unknown) sub-extensive transients 21, 24, i.e., transients that diminish slower than the bulk, $\omega<1$, which at times obscure the physical interpretation to a point of arbitrariness [25]. Even in mean-field, exact results for scaling properties of the glassy phase at short of the thermodynamic limit are few [23, 44,47]. Finding an accessible problem as a model to make conceptual inroads on determining FSC would thus constitute a major advance for RSB.

Numerical simulation, in fact, have provided numerous insights into the nature of FSC in Ising spin-glass models. It was found that ground state energies (and entropies) for mean-field systems of $N$ spins have FSC decaying to excellent approximation with $N^{-\frac{2}{3}}$. This was observed first for BL with bimodal bonds [36, 37] and subsequently [41-43, 48] also for SK. (BL with Gaussian bonds exhibit FSC with $\omega \approx 0.8$ [40, 49].) For finite-dimensional Ising spin glasses (EA), FSC-collapse of domain-wall excitations at $T \rightarrow 0$ allowed an accurate determination of the stiffness exponent $\theta$ in dimensions $d=3, \ldots, 7$ [50]. This exponent is fundamental to many aspects of the glassy state [3, for instance, $\theta\left(d_{l}\right)=0$ defines the lower critical dimension, which appears close to $d_{l}=2.5$ [22, [51, 52], while its determination for $d \geq 6$ allowed a direct check
Table I: List of the fitted values for the average ground state energies $\left\langle e_{0}\right\rangle_{N=\infty}$, the correction amplitude $A$, and the FSC exponent $\omega$ of the SK model at various bond-densities $p$, obtained by fitting the numerical data displayed in Fig. 2 to the asymptotic form in Eq. 22. That fit was conducted over the specified range of system sizes $N$.

\begin{tabular}{l|lccc}
\hline \multicolumn{1}{c|}{$p$} & \multicolumn{1}{c}{$\left\langle e_{0}\right\rangle_{\infty}$} & $\omega$ & $A$ & $N$-range \\
\hline \hline 0.005 & $-0.751(1)$ & $1.39(1)$ & $448(5)$ & $340-2047$ \\
0.01 & $-0.752(1)$ & $1.32(1)$ & $125(5)$ & $165-2047$ \\
0.02 & $-0.755(1)$ & $1.16(1)$ & $26(3)$ & $255-1023$ \\
0.03 & $-0.757(1)$ & $1.02(1)$ & $9(1)$ & $180-512$ \\
0.05 & $-0.761(1)$ & $0.86(1)$ & $3.3(5)$ & $165-512$ \\
0.1 & $-0.762(1)$ & $0.79(1)$ & $1.7(1)$ & $63-1023$ \\
0.2 & $-0.762(1)$ & $0.73(1)$ & $1.04(7)$ & $63-1023$ \\
0.3 & $-0.762(1)$ & $0.71(1)$ & $0.91(5)$ & $63-1023$ \\
0.4 & $-0.762(1)$ & $0.70(1)$ & $0.86(5)$ & $63-1023$ \\
0.5 & $-0.762(1)$ & $0.69(1)$ & $0.80(4)$ & $45-1023$ \\
0.6 & $-0.762(1)$ & $0.68(1)$ & $0.75(3)$ & $45-1023$ \\
1.0 & $-0.76323(5)$ & $0.666(3)$ & $0.71(1)$ & $80-2047$ \\
\hline
\end{tabular}

on mean-field predictions [23]. In particular, FSC were shown to decay consistently as in Eq. 22, applied to hyper-cubic lattices of size $N=L^{d}$ with $\omega=1-\theta / d[24$, suggesting the importance of domain-wall excitations for FSC [21. Recently, we have proposed to use FSC analysis to assess the quality and scalability of optimization heuristics for hard combinatorial problems [14].

In this study, we generate $N \times N$ symmetric bond matrices with entries from a dilute bond-distribution

$$
P(J)=p \delta\left(J^{2}-\frac{1}{p N}\right)+(1-p) \delta(J),
$$

such as to minimize the SK-Hamiltonian [1],

$$
H_{J}=-\sum_{i>j} J_{i j} \sigma_{i} \sigma_{j}
$$

over the set of $N$ Ising spin variables, $\sigma_{i}= \pm 1$. We thus approximate the ground state energy density, $e_{0}(N, p)=$ $\frac{1}{N} \min _{\vec{\sigma}} H_{J}$, for each instance $J$. Like for SK at $p=1$, $\stackrel{N}{P}(J)$ is symmetric with variance $\left\langle J^{2}\right\rangle=1 / N$ but higher moments for the dilute SK diverge for $p \rightarrow 0$, i.e., $\left\langle J^{2 n}\right\rangle=p /(p N)^{n}$ for $n=2,3, \ldots$ [62]. For each bonddensity $p(0<p \leq 1)$, we sample ensemble averages $\left\langle e_{0}\right\rangle_{N}$ of the ground state energies over a range of sizes $N$, where $P(J)$ in Eq. (3) ensures that the average thermodynamic ground state energy is universal [8, 53, $\left\langle e_{0}\right\rangle_{\infty}=e_{\text {Parisi }}=-0.7631667265 \ldots$, first approximated by Parisi [4]. Here, we report on the results for a range of values $p<1$ and find surprisingly non-trivial behavior in the continuous dependence of $\omega(p)$. As the topology of the diagram in Fig. 11 suggests, RSB should remain in effect for all $p$, possibly even in the limit $p \rightarrow 0$, where a solution should become trivial. (Even for the smallest 


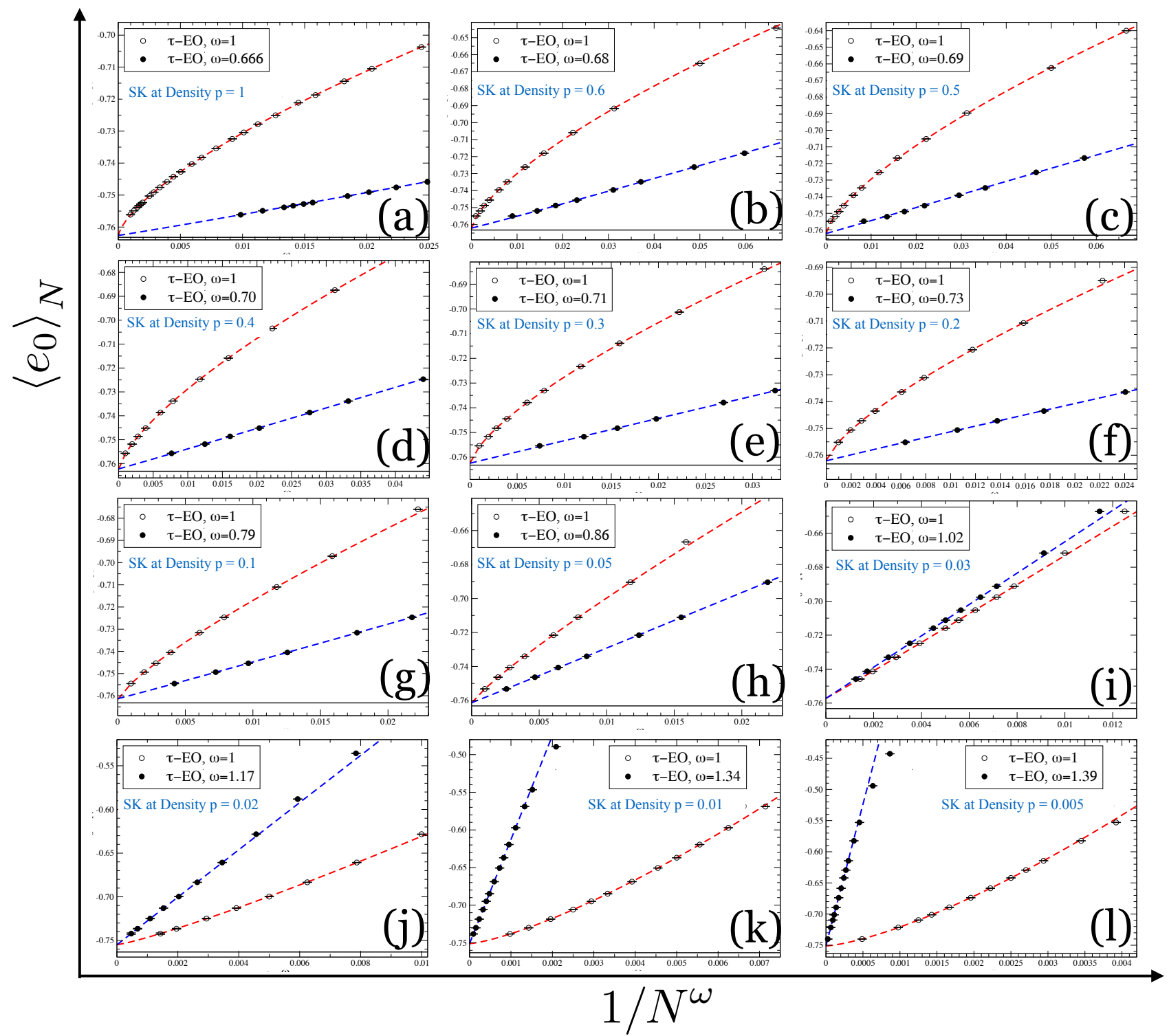

Figure 2: Extrapolation for the rescaled ground-state energy densities, $\left\langle e_{0}\right\rangle_{N}$, of the diluted SK model of bond-density $p$ at different sizes $N$, where each data point is plotted once for $1 / N$ (i.e., $\omega=1$, open symbols) and a second time for $1 / N^{\omega}$ with a value of $\omega$ chosen such that the extrapolation to the thermodynamic limit at the intercept $1 / N^{\omega} \rightarrow 0$ is asymptotically linear (closed symbols). Each panel (a-l) depicts a different density $p$, where data is fitted to the asymptotic form in Eq. (2) (drawn as either red or blue-dashed lines, resp.)Each fit obtains the exponent $\omega$ and the thermodynamic ground-state energy density $\left\langle e_{0}\right\rangle_{N=\infty}$, which should approach the Parisi energy density, $e_{\text {Parisi }}=\left\langle e_{0}\right\rangle_{N \rightarrow \infty}$, for all $p$ [53] (horizontal line), listed in Tab. I]

constant $p$, there is a neighborhood of the thermodynamic limit, for sizes $\frac{1}{p} \ll N<\infty$, where the dilute system is dense enough to be above the percolation transition for sparse random graphs at $c=1$, i.e., $p_{c} \sim 1 / N$ [26], see Fig. 1.)

The following results are obtained with the Extremal Optimization heuristic (EO) [54 [56]. For a generic combinatorial optimization problem, EO performs a local search [56, 57] on an existing configuration of $N$ variables by changing preferentially those of poor local ar- rangement. For example, in case of the spin glass model in Eq. (4), it assigns to each spin variable a "fitness" $\lambda_{i}=\sigma_{i} \sum_{j=1}^{N} J_{i, j} \sigma_{j}$, corresponding to the negative of the local energy of each spin, so that $H=-\frac{1}{2} \sum_{i=1}^{N} \lambda_{i}$ reproduces the Hamiltonian for SK in Eq. (4). A local search with EO requires the ranking of these fitnesses $\lambda_{i}$ from worst to best, $\lambda_{\Pi(1)} \leq \lambda_{\Pi(2)} \leq \ldots \leq \lambda_{\Pi(N)}$, where $\Pi(k)=i$ is the index for the $k^{\text {th }}$-ranked variable $\sigma_{i}$. In the basic version of EO, it always updates the lowest rank, $k=1$ [54, 58, 59]. Instead, $\tau-\mathrm{EO}$ as used here se- 


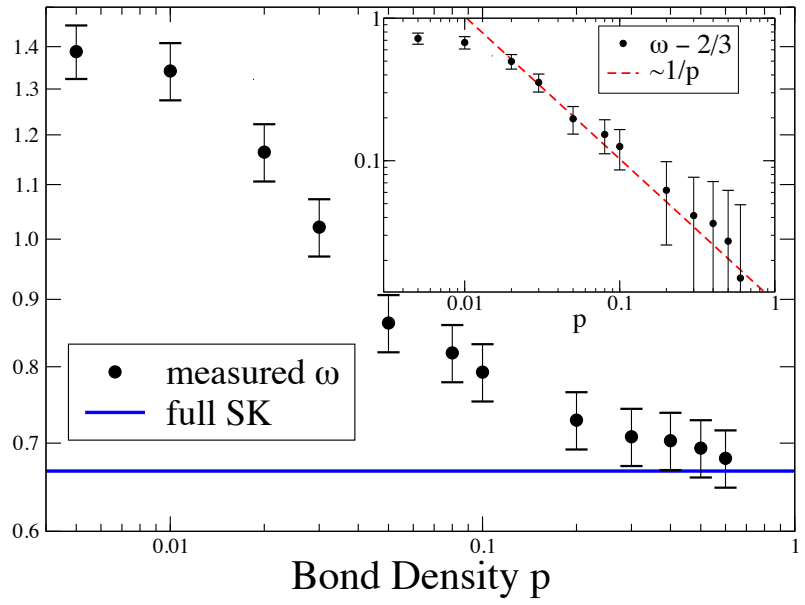

Figure 3: Plot of the fitted values for the exponent $\omega$ controlling the FSC in the extrapolation of the ground state energies shown in Fig. 2 for the bond-diluted SK model as a function of bond-density $p$. The date for $\omega$ can be found in Tab. I. Inset: Except for the smallest values of $p$, the exponent subtracted by its value for $\mathrm{SK}\left(\omega_{\mathrm{SK}} \approx \frac{2}{3}\right.$ at $\left.p=1\right)$, i.e., $\omega-\frac{2}{3}$, appears to approach the SK-value roughly hyperbolically, $\sim 1 / p$.

lects the $k^{\text {th }}$-ranked variable with a scale-free probability $P_{k} \propto k^{-\tau}$. The selected variable is updated unconditionally, and its fitness and that of its neighboring variables are reevaluated. This update is repeated as long as desired, where the unconditional update ensures significant fluctuations, yet, sufficient incentive to return to nearoptimal solutions due to selection against variables with poor fitness, for the right choice of $\tau$. Clearly, for finite $\tau$, EO never "freezes" into a single configuration; it instead records one (or even an extensive set [37, 60]) of the best configurations in passing. Our specific implementation of $\tau-\mathrm{EO}$ for SK proceeds is described in Ref. [42].

EO is implemented [63] for denser instances $(p \geq 0.05)$ as described in Refs. [41, 42, for sparser instances $(p \leq$ 0.05) as described in Refs. [36, 37]; we have obtained statistically identical results for both at $p=0.05$. For any given value of $p$, we generate a large number of instances over a large range of sizes $N$ (from $10^{5}$ instances for all $N<200$ to $2 \times 10^{3}$ at $N \approx 1000$, to $10^{2}-10^{3}$ for $N>1000)$ and average the obtained ground-state energies, $\left\langle e_{0}\right\rangle_{N}$, plotted as a function of $N$ in Fig. 22 l. It is well-known that finding solutions of lowest energy for each instance corresponds an NP-hard combinatorial problem (Max-Cut [61]), and a significant effort must be undertaken to minimize systematic errors in the approximation of ground states. Luckily, we can gauge the accuracy of EO (and any other heuristic [14]) using the theoretical predictions already obtained with RSB. For instance, in panel (a) of Fig. 2, pertaining to SK $(p=1)$ as previously studied in Ref. [41, the EO data was extrapolated to the thermodynamic limit and fit ac- cording to Eq. 22 to reproduce the RSB prediction for $e_{\text {Parisi }}$ to 5 digits of accuracy. Similarly, EO applied to sparse networks [36, 37] reproduced the RSB prediction for BL of fixed degree $c=3$ from Ref. [27] to 4 digits of accuracy. Further application of EO to BL of fixed degrees $c=4, \ldots, 26$ provided predictions for thermodynamic $\left\langle e_{0}^{(c)}\right\rangle_{N=\infty}$, which themselves extrapolate consistently for $c \rightarrow \infty$ such that $c^{-\frac{1}{2}}\left\langle e_{0}^{(c)}\right\rangle_{\infty} \sim e_{\text {Parisi. }}$. Thus, the extrapolation plot, i.e., the very fact that a scaling according to Eq. (2) can be consistently applied, becomes a bootstrap measure of validation in its own right [14].

In Tab. I we list all parameters obtained from the data displayed in Fig. 2 for each value of $p$ asymptotically for large $N$ to Eq. (2). We observe that the dependence of the FSC exponent $\omega$ on $p$, shown in Fig. 3, is quite remarkable. While SK [41,43, 48, as well as sparse networks [21, 36, 37] with bimodal bonds have consistently exhibited FSC with $\omega \approx \frac{2}{3}$, independent of degree $c$, for fixed $p<1$ in the dilute SK we find significant variation in $\omega(p)$. For decreasing $p, \omega(p)$ rises from its SK-value at $p=1$ with what appears to be a continuous hyperbolic form, roughly $\omega-\frac{2}{3} \sim \frac{1}{p}$, for about two decades, $0.03 \leq p \leq 1$, as the inset of Fig. 3 suggests. Leaving $\left\langle e_{0}\right\rangle_{\infty}$ as a fitting paramters, the exact result, $\left\langle e_{0}\right\rangle_{\infty}=e_{\text {Parisi }}$, is reproduced within errors for $p>0.03$, see Tab. I. Significant deviations only arise for the smallest values of $p$ studied here, and it is not obvious whether these are due to systematic errors in EO or due to the assumptions underlying Eq. (2) Fixing $\left\langle e_{0}\right\rangle_{\infty}=e_{\text {Parisi }}$ for the fit has virtually no effect on our key result, the variation of $\omega(p)$, as listed in Tab. If for $p>0.03$. However, the data for smaller $p$ no longer fit to any FSC we considered, such as higher order corrections to Eq. (2), logarithmic corrections, etc., unless we assume large systematic errors and discount EO-data for larger $N$. Not only does that contradict aforementioned results in Refs. [36, 37, it renders any such fit arbitrary. It is interesting that this transition occurs at a value of $p$ where the fitted value of $\omega(p)$ just about passes unity, suggesting that a bulk $(1 / N)$ correction, subdominant in Eq. (2) for $\omega<1$, might interfere. (At $p=1$, such a correction has been ruled out for SK in Ref. 41.).) The breakdown of simple FSC in Eq. (2) is also signaled by the diverging amplitude $A(p)$ in Tab. I.

An analytic study of the dilute SK model in the limit of $p \rightarrow 0$ should be able to reveal whether the limit for $\omega$ is regular or singular. A perturbative expansion around that limit might also shed light on the nature of FSC in RSB, since there does not appear to be a transition at any finite $p$ from RSB near $p=1$ to a simple replicasymmetric phase, at least at $T=0$. Thus, future studies should explore the properties of the dilute SK for finite $T$. But even at the ground-state level, we intend to explore the behavior of other characteristic features, such as the ensemble fluctuations in the ground state ener- 
gies [23, 41, 44]. As there is expected to be a scaling relation between the FSC-exponent $\omega$ and the exponent describing such fluctuations [21, investigating their relation while evolving with $p$ should be very revealing about the nature of universality at $T=0$.

* Electronic address: sboettc@emory.edu

[1] D. Sherrington and S. Kirkpatrick, Phys. Rev. Lett. 35, 1792 (1975).

[2] S. F. Edwards and P. W. Anderson, J. Phys. F 5, 965 (1975).

[3] K. H. Fischer and J. A. Hertz, Spin Glasses (Cambridge University Press, Cambridge, 1991).

[4] G. Parisi, Phys. Rev. Lett. 43, 1754 (1979).

[5] G. Parisi, J. Phys. A 13, 1101 (1980).

[6] M. Mézard, G. Parisi, and M. A. Virasoro, Spin glass theory and beyond (World Scientific, Singapore, 1987).

[7] M. Talagrand, Spin Glasses: Cavity and Mean Field Models (Springer, Berlin, 2003).

[8] D. Panchenko, J. Stat. Phys. 149, 362 (2012).

[9] D. L. Stein and C. M. Newman, Spin Glasses and Complexity (Princeton University Press, Princeton, 2013).

[10] J. J. Hopfield, PNAS 79, 2554 (1982).

[11] E. Schneidman, M. J. Berry, R. Segev, and W. Bialek, Nature 440, 1007 (2006).

[12] M. Mezard, G. Parisi, and R. Zecchina, Science 297, 812 (2002).

[13] M. Mézard and A. Montanari, Constraint Satisfaction Networks in Physics and Computation (Oxford University Press, Oxford, 2006).

[14] S. Boettcher, Phys. Rev. Res. 1 (2019).

[15] D. J. Wales, Energy landscapes (Cambridge University Press, Cambridge, 2003).

[16] S. A. Kauffman and E. D. Weinberger, J. Theor. Bio. 141, 211 (1989).

[17] R. Axelrod, The Complexity of Cooperation (Princeton University Press, 1997), ISBN 0691015678.

[18] H. Nishimori, Statistical Physics of Spin Glasses and Information Processing (Oxford University, Oxford, 2001).

[19] R. Oppermann, M. J. Schmidt, and D. Sherrington, Phys. Rev. Lett. 98, 127201 (2007).

[20] R. K. Pathria, Statistical Mechanics, 2nd Ed. (Butterworth-Heinemann, Boston, 1996).

[21] J.-P. Bouchaud, F. Krzakala, and O. C. Martin, Phys. Rev. B 68, 224404 (2003).

[22] S. Boettcher, Phys. Rev. Lett. 95, 197205 (2005).

[23] G. Parisi and T. Rizzo, Phys. Rev. Lett. 101, 117205 (2008).

[24] S. Boettcher and S. Falkner, EPL (Europhysics Letters) 98, 47005 (2012).

[25] S. Boettcher, Philosophical Magazine 92, 34 (2012).

[26] B. Bollobas, Random Graphs (Academic Press, London, 1985).

[27] M. Mézard and G. Parisi, Eur. Phys. J. B 20, 217 (2001).

[28] G. Kotliar, P. W. Anderson, and D. L. Stein, Phys. Rev. B 27, R602 (1983).

[29] H. G. Katzgraber and A. P. Young, Phys. Rev. B 67, 134410 (2003).

[30] S. Boettcher, H. G. Katzgraber, and D. Sherrington, J.
Phys. A: Math. Theor. 41, 324007 (2008).

[31] H. G. Katzgraber, D. Larson, and A. P. Young, Phys. Rev. Lett. 102 (2009).

[32] T. Aspelmeier, W. Wang, M. A. Moore, and H. G. Katzgraber, Phys. Rev. E 94, 022116 (2016).

[33] Y. T. Fu and P. W. Anderson, J. Phys. A: Math. Gen 19, 1605 (1986).

[34] L. Zdeborova and S. Boettcher, J. Stat. Mech. P02020 (2010).

[35] M. N. Barber, in Phase Transitions and Critical Phenomena, edited by C. Domb and J. L. Lebowitz (Academic Press, New York, 1983), vol. 8, p. 146.

[36] S. Boettcher, Phys. Rev. B 67, R060403 (2003).

[37] S. Boettcher, Euro. Phys. J. B 31, 29 (2003).

[38] M. Palassini and A. P. Young, Phys. Rev. Lett. 85, 3017 (2000).

[39] I. A. Campbell, A. K. Hartmann, and H. G. Katzgraber, Phys. Rev. B 70, 054429 (2004).

[40] S. Boettcher, Euro. Phys. J. B 74, 363 (2010).

[41] S. Boettcher, J. Stat. Mech. 2010, P07002 (2010).

[42] S. Boettcher, Eur. Phys. J. B 46, 501 (2005).

[43] T. Aspelmeier, A. Billoire, E. Marinari, and M. A. Moore, J. Phys. A: Math. Theor. 41, 324008 (2008).

[44] G. Parisi and T. Rizzo, Phys. Rev. B 79, 134205 (2009).

[45] G. Parisi and T. Rizzo, J. Phys. A: Math. Theor. 43, 045001 (2010).

[46] G. Parisi, F. Ritort, and F. Slanina, J. Phys. A 26, 247 (1993).

[47] G. Parisi, F. Ritort, and F. Slanina, J. Phys. A 26, 3775 (1993).

[48] S.-Y. Kim, S. J. Lee, and J. Lee, Phys. Rev. B 76 (2007).

[49] G. Parisi, L. Sarra, and L. Talamanca, J. Stat. Mech. 2019, 033302 (2019).

[50] S. Boettcher, Europhys. Lett. 67, 453 (2004).

[51] S. Franz, G. Parisi, and M. A. Virasoro, J. Phys. I (France) 4, 1657 (1994).

[52] A. Maiorano and G. Parisi, Proceedings of the National Academy of Sciences 115, 5129 (2018).

[53] P. Carmona and Y. Hu, Ann. Inst. H. Poincare Probab. Statist. 42, 215 (2006).

[54] S. Boettcher and A. G. Percus, Artificial Intelligence 119, 275 (2000).

[55] S. Boettcher and A. G. Percus, Phys. Rev. Lett. 86, 5211 (2001).

[56] A. Hartmann and H. Rieger, eds., New Optimization Algorithms in Physics (Wiley-VCH, Berlin, 2004).

[57] H. H. Hoos and T. Stützle, Stochastic Local Search: Foundations and Applications (Morgan Kaufmann, San Francisco, 2004).

[58] S. Boettcher, Computing in Science and Engineering 2, 75 (2000).

[59] J. Brownlee, Clever Algorithms: Nature-Inspired Programming Recipes (LuLu, 2011).

[60] S. Boettcher and A. G. Percus, Phys. Rev. E 69, 066703 (2004).

[61] M. R. Garey and D. S. Johnson, Computers and Intractability: A Guide to the Theory of NP-Completeness (W. H. Freeman, New York, 1979).

[62] As pointed out by a referee.

[63] See also the demo at http://www.physics.emory.edu/faculty/boettcher/Research/EO_demo/demoSK.c 